\title{
Claudio Guillén en Harvard, génesis del comparatismo español ${ }^{*}$
}

\section{Claudio Guillén at Harvard, Genesis of Spanish Comparatism}

\author{
Antonio Martín Ezpeleta \\ Universidad de Valencia \\ anmarez@uv.es
}

ORCID iD: https://orcid.org/0000-0003-0210-3399

\section{RESUMEN}

En el presente trabajo se reconstruye el paso de Claudio Guillén por la Universidad de Harvard, donde se formó como comparatista junto a varios de los mejores estudiosos del momento, y donde ejerció como profesor durante sus últimos años en Estados Unidos. Esta revisión de la trayectoria profesional y personal de Claudio Guillén ayuda a entender la evolución de la disciplina de la Literatura Comparada en la Universidad de Harvard dentro del marco del comparatismo norteamericano de postguerra, los estudios literarios del propio Claudio Guillén y su esfuerzo por impulsar la disciplina de la Literatura Comparada en España, así como la particular circunstancia social de los intelectuales españoles y sus familias en el exilio. Para todos estos asuntos, la documentación administrativa y personal custodiada en los Archivos de Harvard resulta fundamental.

Palabras Clave: Claudio Guillén; Harvard; Literatura Comparada; hispanistas; exilio.

\section{ABSTRACT}

This essay seeks to reconstruct Claudio Guillén's life at Harvard University, where he was educated as a comparatist along with some of the best scholars at that time, and where he taught his last years in the United States. This review of the professional and personal trajectories of

* Este trabajo forma parte de un proyecto de investigación sobre la historia del Departamento de Lenguas y Literaturas Romances de la Universidad de Harvard, que se benefició de una beca MEC/Fulbright desarrollada en dicha institución. Agradezco al profesor Luis Fernández Cifuentes sus amables apreciaciones. La documentación inédita que aquí reproduzco se publica con el permiso expreso de la Houghton Library de la Universidad de Harvard y la autorización pertinente de la viuda y heredera de Claudio Guillén, la señora Margarita Ramírez, de cuya amabilidad quiero aquí dejar testimonio. 
Claudio Guillén helps us to understand the evolution of the discipline of Comparative Literature at Harvard University within the framework of postwar American comparatism, the literary studies of Claudio Guillén himself and his effort to promote the discipline of Comparative Literature in Spain, as well as the particular social circumstances of Spanish intellectuals and their families in exile. For all these matters, the administrative and personal documentation kept in the Harvard Archives is essential.

Key words: Claudio Guillén; Harvard; Comparative Literature; Hispanists; Exile.

Uno de los asuntos fundamentales que hay que tener en cuenta a la hora de abordar la disciplina de la historia de la crítica literaria es la historia de sus instituciones. Este es precisamente el contexto donde se enmarca el presente trabajo que pretende revisar el paso de Claudio Guillén (1924-2007), por la Universidad de Harvard, en cuyos departamentos de Literatura Comparada y Lenguas y Literaturas Romances se formó e impartió clases dejando una indeleble huella, como ambos departamentos reconocieron en unas jornadas celebradas en su memoria en febrero de 2008 al poco de fallecer ${ }^{1}$. Por otro lado, el lector de las obras de Claudio Guillén sabe de la fidelidad del comparatista a dicha institución -que le concedió una beca para iniciarse en los estudios de Literatura Comparada-y a muchos de sus profesores y colegas, quienes no solo marcaron su biografía intelectual sino también personal, como reflejan las dedicatorias de sus libros (a Harry Levin, Renato Poggioli, Amado Alonso o Stephen Gilman) o los textos que el discípulo redactó sobre el recuerdo de sus maestros, que más adelante reaparecerán.

Así pues, en las siguientes páginas se esbozan las dos caras del alumno y el profesor Claudio Guillén en Cambridge, asunto que, como queda dicho, supondrá relacionar al autor de Entre lo uno y lo diverso con grandes figuras como las mencionadas o, entre otras, la del mejor amigo de su padre, Pedro Salinas, que fue un gran referente para Claudio Guillén. Además de su relación con Harvard, también se relata brevemente su paso por los excelentes cursos de verano de la Universidad de Middlebury a los que asistió el joven estudiante. En estos cursos aprendió con varios de los mejores profesores españoles, pero también le resultaron fundamentales a la hora de identificarse y confraternizar con los intelectuales del exilio republicano en Estados Unidos, de cuyo grupo formó parte en calidad de miembro de la segunda generación ${ }^{2}$.

${ }^{1}$ En ellas participaron Donald Fanger (el sucesor de Claudio Guillén en la Cátedra Harry Levin), Ellen Lokos (que fue alumna de Claudio Guillén) y Francisco Márquez Villanueva (colega de departamento de Claudio Guillén), a quien agradezco en un sentido homenaje póstumo la copia del texto que allí leyó, así como sus inteligentes comentarios sobre mi trabajo.

2 Sobre Claudio Guillén contamos con varios estudios monográficos como los de García Jurado, Reader y Villar 2009, y los más recientes de Monegal, Bou y Cots 2017 o Cots y Monegal 2010. 


\section{El EXILIO A Estados UNidos Y EL DESARROLlo DE UNA VOCACIÓN INTELECTUAL}

En esta segunda generación se encontraba incluido también Jaime Salinas, quien compartió con Claudio Guillén la amistad y diversos avatares de sus vidas, aunque no tantos como sorprendentemente se encuentran al cotejar las biografías de sus respectivos padres. Resulta interesante, según ha hecho el propio Jaime Salinas en sus memorias Travesías (2003), reflexionar sobre estos primeros años en Estados Unidos de los jóvenes Jaime y Claudio. Recuérdese que ambos, hijos de madres francesas y padres españoles, eran bilingües al llegar a Norteamérica (al poco tiempo políglotas) y los dos también habían cursado el prestigioso sistema educativo francés en Europa. Sin embargo, en Estados Unidos siguieron caminos educativos diferentes, algo que, como afirma Jaime Salinas (2003, 125), bifurcó en cierto modo sus vidas: Claudio Guillén pudo reanudar sus estudios en el sistema francés en el Lycée de Montreal, pero Jaime Salinas tuvo que seguir la educación americana, menos exigente económicamente.

En efecto, Claudio Guillén siguió formándose de manera notable terminando su bachillerato francés (tuvo que examinarse en Nueva York de las últimas pruebas) y así pudo acceder directamente a los colleges americanos en el segundo año. Esto sucedió en el estado de Massachusetts, donde tras haber estado en Canadá el curso de 1939-1940 la familia Guillén se mudó para que Jorge Guillén ocupara la plaza en Wellesley College que dejó Pedro Salinas. La carrera que estudió en Williams, un prestigioso College cercano a Wellesley donde fue becario, fue Literatura Inglesa ${ }^{3}$. Sin embargo, sus estudios se vieron interrumpidos por la Segunda Guerra Mundial, acontecimiento en que valientemente decidió entrar a formar parte como voluntario en el ejército del General De Gaulle en $1943^{4}$. Una vez concluido su servicio en 1946 regresó a Es-

${ }^{3}$ He podido averiguar, gracias a la amabilidad de las bibliotecarias Linda Hall y su ayudante Stella Onochie de Williams College, los profesores -ninguno especialmente conocido- que Claudio Guillén tuvo durante sus estudios en este centro durante los años 19431944 (en los archivos consta que se graduó en bachelor of Arts con el curso de 1944, no obstante fue en 1943 Claudio Guillén 2001, 22; Guillén y Salinas 1992, 311): John Hawley Roberts, Samuel Edward Allen, Hallet D. Smith, Nelson Sherwin Bushnell, Rowland Hill Evans, Williams Merriam Gibson, James Clay Hunt, Roy Lamson, James Elder Michael, Fred Holly Stocking, Williams Graves Perry, Russell Jack Smith, Robert Joseph Allen, Luther Stearns Mansfield, Williams Shepard Dix y Warren Douglas. De todos sus profesores en Williams, Claudio Guillén destacó la admiración que profesaba por Lane Faison (no consta en la lista anterior), historiador del arte de gran vocación docente que asegura Guillén le inició en el ejercicio de relacionar diferentes asuntos y disciplinas (2001, 20-21).

${ }^{4}$ Las noticias sobre este episodio pueden rastrearse en la impagable correspondencia que intercambiaron los poetas Jorge Guillén y Pedro Salinas (Guillén y Salinas 1992, 293, $323,375)$. 
tados Unidos -no sin ciertos apuros burocráticos para formalizar su nueva entrada al país-, donde empezó sus estudios de postgrado en Harvard. Escribe sobre este momento Claudio Guillén en su interesante Entre el saber y el conocer:

Volví a mis estudios después [de la guerra] sin vacilación, con las tremendas ganas, como os podréis imaginar, y la capacidad de trabajo que infunde el haberle visto la cara a la guerra y con ella la podredumbre de la vida militar. Tuve la buena fortuna de ser admitido como alumno de doctorado en la Universidad de Harvard.

A fines de la década de los 40 y durante los 50 se encontraban en Harvard numerosos profesores muy eminentes - escapados, algunos, de las dictaduras europeas. Así el gran helenista Werner Jaeger, o el germanista Karl Viëtor, o el francés Jean Seznec, con quien estudié, finísimo conocedor del Renacimiento, y el norteamericano F.O. Mathiessen, que también fue mi profesor, y el shakespeariano Theodore Spencer. Era natural que en tal ambiente decidiera doctorarme en el Departamento de Literatura Comparada $(2001,22)$.

Harvard becó a Claudio Guillén para que cursara un máster de Literatura Comparada ${ }^{5}$, aunque después decidió continuar estudiando y doctorarse en esta misma disciplina. Explica su padre sobre el ánimo de su hijo en carta de octubre de 1946 a Salinas: «Claudie [Claudio Guillén], muy excitado por su vida en Harvard (definitivamente, me parece, entregado a su sino de intelectual)» (Guillén y Salinas 1992, 402). Pero conviene detenerse ahora brevemente en describir la historia del Departamento de Literatura Comparada de Harvard y sus cursos.

Harry Levin, uno de los impulsores de este departamento, destaca en una suerte de historia de la Literatura Comparada en Harvard que esta disciplina comenzó como tal en 1904, cuando se configuró una línea comparatista de tutela de tesis, y que la fecha de 1906 es la que marca el hito administrativo de la creación del Departamento de Literatura Comparada al separarse del de Lenguas Romances (1994, 14-15). Pero la Literatura Comparada estaba muy presente en Harvard desde hacía más de una docena de años. Y es que la aparición de esta data de la temprana fecha de 1890-1891 ${ }^{6}$, curso académico en

5 En una carta datada el 16 de febrero de 1946 Jorge Guillén explica a Pedro Salinas que su conocido William Berrien, por aquel entonces director del Departamento de Lenguas y Literaturas Romances, tenía muy buena impresión de Claudio Guillén (Guillén y Salinas 1992, 375).

${ }^{6} \mathrm{La}$ información administrativa sobre los departamentos de Literatura Comparada y Lenguas y Literaturas Romances se halla restringida por la conocida como ley de los cincuenta años, es decir, que no se pueden consultar documentos de menos de esa antigüedad. No obstante los bibliotecarios del Archivo de Harvard, cuya ayuda me apresuro a agradecer, sí me han permitido el acceso a una serie de publicaciones periódicas del Presidente de Harvard y la Facultad de Artes y Ciencias donde hay información administrativa sobre dichos 
que se ofertaron dos asignaturas comparatistas impartidas por el profesor Marsh ${ }^{7}$. A partir de aquí el Departamento de Literatura Comparada se fue consolidando paulatinamente al lado del de Filología Comparativa -circunscrito al de Lingüística- y los diferentes departamentos de Lenguas Clásicas y Modernas.

Sin embargo, mientras Claudio Guillén se encuentra estudiando su carrera en Williams, el Departamento de Literatura Comparada de Harvard desapareció temporalmente. Esto fue debido a la participación de Estados Unidos en la Segunda Guerra Mundial y el consecuente debilitamiento de ciertas disciplinas por la falta de alumnos principalmente, así como la tendencia extendida a convertir los estudios de Letras en cursos rápidos de preparación para los jóvenes llamados a ocupar mandos militares que precisaran cierta cualificación ${ }^{8}$.

En el curso de 1946-1947 reapareció la Literatura Comparada en Harvard9, aunque algo mermada de asignaturas y profesores especializados todavía. Harry Levin $(1994,21-22)$ brinda el dato de que hasta esa fecha se habían leído bastantes tesis doctorales comparatistas, pero que estas crecieron exponencialmente a partir de 1949. Esta es la fecha que marca el principio de la edad dorada del departamento y la Literatura Comparada, en palabras de Claudio Guillén $(2005,12)$, quien hizo coincidir en el controvertido prólogo de la segunda edición de Entre lo uno y lo diverso ${ }^{10}$ el declive de la disciplina con la fecha

departamentos (signaturas HUC 8500.16, HU 30.10, HUE 22.153.75), así como a unas fotos de los años 80 del profesor Claudio Guillén charlando amigablemente con colegas de Harvard (Archives accession 17478). (Algunas fotos y noticias de no demasiada importancia a los efectos de este trabajo se pueden hallar también en la publicación periódica de Harvard The Crimson, cuyos archivos están digitalizados y accesibles en: <http://www.thecrimson. com/archives.aspx $>$ ).

${ }^{7}$ La noticia del nacimiento de esta disciplina se encuentra en el informe anual del presidente de la Facultad de Artes y Ciencias (Annual reports of the President 1892, 6). Las asignaturas ofertadas en cuestión, para undergraduates y graduates, eran: «The History of Classical Learning in Europe from the Fifth to the Fifteenth Century, considered with regard to the relation of modern to ancient culture», "Comparative European Literature in the Middle Ages, with especial reference to France and her influence», así como una línea de tutela de tesis $(1892,52)$.

${ }^{8}$ El caso lo explica con detalle Jaime Salinas al referirse a la Universidad de Johns Hopkins, donde su padre Pedro Salinas impartía clases por aquellos años $(2003,147)$.

9 En el informe anual del curso 1945-1946 del presidente de la Facultad de Artes y Ciencias se explica el impulso que pretende darse al Departamento de Literatura Comparada en próximos años: «The development of sound critical standards in literature by means of an active program under the heading of Comparative Literature is a task of equal difficulty. We hope that over a period of years the development of such standards by the rigorous comparison of the different kinds of literature will be at least a possibility. If we succeed, the effect on the teaching of the literatures, and perhaps also the arts, will be national in scope» (Report of the President of Harvard 1948-1949, 38-39).

10 Sobre este prólogo, intitulado «La Literatura Comparada y la crisis de las Humanidades», véase el trabajo de Fernando Cabo Aseguinolaza (2007), que puntualiza la relevan- 
de 1985 y el nacimiento de nuevas corrientes crítico-literarias. No obstante, volvamos al principio de esta edad de oro (el periodo de formación de Claudio Guillén), que vino impulsada por la llegada de algunos grandes profesores que inmediatamente aparecerán, y que convirtió al de Harvard en uno de los departamentos de Literatura Comparada más punteros de Estados Unidos y el mundo.

\section{LA FORMACIÓN COMPARATISTA EN LA UNIVERSIDAD DE HARVARD}

En unos cuadernillos que la Universidad de Harvard publicaba para informar de los diferentes estudios que ofertaba, se pueden recuperar las asignaturas que componían cada curso y programa de doctorado ${ }^{11}$. Los cursos para los graduates eran realmente ambiciosos, y abarcaban desde el estudio del teatro a partir de Esquilo hasta Ben Jonson o desde Racine a Shaw, pasando por la crítica literaria en Francia e Italia durante el Renacimiento y los orígenes y el desarrollo del Romanticismo europeo. Los doctorandos debían también contar con la supervisión de uno de los miembros del Commitee on Comparative Literature, que guiaría sus pasos hasta la redacción de la tesis doctoral. En este se incluía a un joven profesor asociado que sería quien en años venideros iba a propiciar fundamentalmente el desarrollo extraordinario del Departamento de Literatura Comparada, el citado Harry Levin ${ }^{12}$, cuyo retrato nos brinda el propio Claudio Guillén:

cia de la Teoría de la literatura en la definición y práctica de la Literatura Comparada.
11 Los cuadernillos que he utilizado, intitulados Official Register of Harvard Universi-
ty. Final Announcement of the Courses of Instruction Offered by the Faculty of Arts and
Sciences, corresponden a los años comprendidos entre 1946 y 1949 . Al tratarse de un cam-
po que no se hallaba en los anteriores cursos, se incluye en el cuadernillo de 1946-1947 una
explicación introductoria sobre la organización de los estudios de Literatura Comparada:
«The field of Comparative Literature is organized so that systematic study of the subjects
and problems common to various ancient and modern languages may be facilitated. The
graduate degrees of Master of Arts and Doctor of Philosophy are granted in Comparative
Literature. Programs leading to such degrees are arranged by the Committee on Compara-
tive Literature, under the Chairmanship of Professor Sherburn, Chairman of the Division of
Modern Languages. In addition to the courses formally listed below, any courses in litera-
ture carrying graduate credit in the departments of Classic, English, Germanic Languages,
Romance Languages, and Slavic Languages may be used to complete programs. Such
courses should, however, be approved by the Committee as forming a part of a coherent
program. Attention is especially called to Romance Philology, Bibliography and Methods of
Research (Professor Morize). Courses in History and Philosophy that contribute to the un-
derstanding of cultural relation may be approved if they concern the student's special aims»
(Official Register of Harvard University 1946-1947, 38).
12 La administración de Harvard no tardó demasiado en darse cuenta del ímpetu y ta-
lento de Harry Levin. En el informe anual del director de la Facultad de Artes y Ciencias 
Era Harry Levin bastante joven aún, pero su figura era absolutamente magistral. Todo un emblema de Harvard, muy conocido desde sus primeros libros sobre James Joyce, o Christopher Marlowe, o Hamlet, y luego su gran estudio de los novelistas europeos del siglo XIX, The Gates of Horn, era excepcionalmente sabio. Parecía que lo había leído todo y en particular que no olvidaba nada, sin desconocer ninguna de las claves de la literatura europea. No he conocido a nadie que dominase hasta tal punto el espacio de la literatura occidental, que es ilimitado. [...] Sus clases tenían la precisión de un ensayo acabado, con mil hallazgos felices y juegos de ingenio [...] como si escribiera hablando. No le faltaban por supuesto ideas, pero sin la vulgaridad de recalcarlas, prefiriendo citarse a sí mismo entre tantos otros. Un compañero mío de clase, Pierre Schneider (hoy famoso crítico de arte en Francia), calificaba sus lecciones de «las alusiones perdidas». (2001, 25-26)

Ya en el curso de 1948-1949 se puede observar la mano de su director, Levin, y el crecimiento y enriquecimiento que este operó en la oferta de cursos, como ahora se describirá. Acaso es menos conocido que Levin otro joven profesor asociado, Renato Poggioli, italiano emigrado especialista en lenguas eslavas, cuyo recuerdo sigue visible hoy en las lectures que llevan su nombre y que Harvard organiza anualmente. Tiempo después Claudio Guillén (2001, 26-27) ha insistido en revalorizar la figura de Poggioli y situarlo al lado de Levin como coartífices de uno de los momentos más fértiles del Departamento de Literatura Comparada de Harvard, que, como se afirmaba más arriba, creció exponencialmente en número de profesores (entre ellos algunos de la talla de Roman Jakobson), asignaturas impartidas y calidad de las mismas. Claudio Guillén tuvo la suerte de vivir este momento y, en consecuencia, ser uno de los jóvenes estudiantes mejor formados en esta materia en aquellos años cincuenta. Veamos ahora cuáles fueron esas asignaturas ofertadas por el departamento y que Claudio Guillén cursó. Antes, sin embargo, hay que apuntar de la mano del propio Guillén cómo estaban planteados los estudios de Literatura Comparada en Harvard:

Se trata solamente de estudios de doctorado, o de lo que aquí llamamos tercer ciclo. Cada doctorando estudia tres literaturas: una principal (major literature) desde la Edad Media hasta nuestros días (tratándose de literaturas modernas), con la necesaria fundamentación histórica y filológica; y otras dos, reducidas a una misma época - por ejemplo el siglo XVIII, o el Renacimiento y Barroco; o los siglos XIX y XX-, conocida a fondo en las lenguas originales. [...] De tal suerte que este doctorando tiene dos especialidades, la una horizontal y la otra vertical. [...] Hay también por supuesto asignaturas nucleares, como una Introducción a la

se hace referencia expresa a este hecho: «Under the leadership of Associate Professor Levin as Chairman, the Department of Comparative Literature very considerably increased its offerings and developed a fruitful cooperation with the experimental courses in Humanities, now given by the Committee on General Education» (Report of the President of Harvard 1948-1949, 45). 
Literatura Comparada, y cursos de Teoría literaria o de Historia de la Poética; así como seguimientos individuales mediante tutorías, que en este terreno son indispensables. [...] Claro está que se exigen conocimientos serios de la literatura griega y latina $(2001,24-25)$.

Efectivamente, desde el principio se había prestado en el Departamento de Literatura Comparada de Harvard gran atención a la tradición clásica, que siguió siendo un pilar maestro del programa en años sucesivos. Desde el curso de 1948-1949, sin embargo, empiezan a aparecer también otras literaturas nacionales que antes no habían sido tenidas en cuenta, como sucede con la rusa. El profesor Poggioli -que ya forma parte del comité académico del departamento- es el responsable, por ejemplo, de impartir una asignatura titulada «Dostoyevsky and Tolstoy». Lo mismo sucede con asignaturas inéditas sobre Crítica literaria y Teoría de la literatura (las más de ellas son responsabilidad de Levin, como por ejemplo «Literature and Society», o Poggioli, «Theory of the Literature») ${ }^{13}$ y estudios monográficos sobre los archigéneros teatro, poesía y prosa en Europa («Allegory», «Satire», «Ideas of Tragedy», «Forms of the Modern Novel», «The Romantic Lyric: English, French, and German»...), así como el seguimiento de movimientos historiográfico-literarios como el «Realismo» ( «German Realism and Naturalism, with special reference to other European influences»...), pero también el «Clasicismo» o el «Simbolismo» (ambos impartidos por Poggioli). Además aparecen cursos monográficos sobre tripletas de genio literarios como «Hardy, Conrad, and Gide», «Proust, Joyce, and Mann», etcétera, o temas como «Goethe's Faust and the Faust Legend», «The Romantic Hero»...

Esta división en formas y géneros, periodos historiográfico-literarios y temas no deja de recordar, con el enriquecimiento consustancial a la disciplina, los capítulos de esa suerte de manual general sobre los estudios de Literatura Comparada que conocemos con el título de Entre lo uno y lo diverso, que Claudio Guillén publicó en 1985 y reformuló someramente en 2005. Su papel de difusor de los estudios de Literatura Comparada en España es de sobra conocido. Pero conviene señalar para terminar esta descripción del Departamento de Literatura Comparada de Harvard que a esta serie de asignaturas citadas se unían otros eventuales cursos impartidos por profesores visitantes de reconocido prestigio, como René Wellek (en 1949-1950) o, entre otros, Northrop Frye (1956-1957), según queda constancia en el Official Register de los años 1946 a 1949.

${ }^{13}$ Escribe Claudio Guillén: «No estaba muy de moda por aquel entonces la Teoría literaria, pero él [Poggioli] conocía íntimamente los principales textos de la Poética y, tal vez gracias a aquel ambiente animado en Italia por las polémicas en torno a Benedetto Croce, y otras muy anteriores, nos explicaba con luminosa claridad las ideas de Vico o de los hermanos Schlegel; y en suma, sabía aunar la sensibilidad del lector de poesía y la inquietud teórica de una manera excepcionalmente viva y verdadera» $(2001,26-27)$. 
En fin, se puede comprobar la riqueza y amplitud de conocimientos, así como el prestigio de los responsables de la docencia en este Departamento de Literatura Comparada. Solo se echa en falta algo de atención a la Literatura Hispanoamericana, que, frente a lo que ocurre en el Departamento de Lenguas y Literatura Romances que poco a poco va imprimiéndole protagonismo - empieza con un curso sobre la Lengua Hispanoamericana impartido por Amado Alonso-, no es apenas considerada durante esos años cuarenta y cincuenta.

En el departamento homólogo de Lenguas y Literaturas Romances, por su parte, cabe destacar la llegada de profesores como Francisco García Lorca, Juan Marichal, Raimundo Lida, Stephen Gilman (estos dos últimos impartieron con regularidad asignaturas en el Departamento de Literatura Comparada) o, entre otros, los profesores invitados por aquellos años: Rafael Lapesa, Dámaso Alonso, Américo Castro y otros eminentes estudiosos. No extrañará, pues, que, con Amado Alonso como principal figura, la sección española del Departamento de Lenguas y Literaturas Romances de Harvard se enriqueciera enormemente ${ }^{14}$.

El trato con profesores españoles le marcó profundamente. Ya desde niño estuvo en contacto con numerosos escritores y críticos amigos de su padre, el poeta y catedrático Jorge Guillén. Sin embargo, fue en esta época de estudiante cuando realmente se sintió profundamente atrapado por ellos y por el quehacer intelectual. Así, pasó de lector a estudioso para, tiempo más tarde, convertirse en profesor, según confiesa el propio autor (2001, 18). El lugar de encuentro más propicio no fue solamente Harvard, sino también la Universidad de Middlebury, que organizaba unos excelentes cursos de verano a los que acudían como profesores los más notables intelectuales españoles exiliados en Estados Unidos y que merecen también cierta atención.

\section{Los Veranos EN La Universidad De Middlebury}

Claudio Guillén ha explicado en un par de ocasiones la importancia que tuvieron para él sus estancias estivales en Middlebury en estos términos:

${ }_{14}$ Claudio Guillén destacó de entre sus grandes maestros romanistas a Amado Alonso (2001, 23-24), así como a Joaquín Casalduero, Américo Castro -que en puridad no fue su maestro- y Pedro Salinas (2001, 28ss.), sobre el que luego se volverá. Como es sabido, Claudio Guillén escribió en diversas ocasiones sobre muchos de sus maestros y colegas de departamento (véase ahora los prólogos a Francisco García Lorca 1948 y Gilman 2002). La mayor parte han sido reelaborados ligeramente en De leyendas y lecciones (2006, 105-249, 283-287) y Entre el saber y el conocer (2001, 11-34), donde confiesa el autor una gran motivación autobiográfica: «No se te ocultará así [lector] la raíz autobiográfica de esta escritura, sobre todo a través de la diferencia entre las piezas escritas en el exilio, rodeado yo de grandes maestros españoles y de compañeros desterrados que compartían una visión esperanzada y bastante idealizada de su país de origen, y lo pensado sentido y luego en España» $(2006,9)$. 
Amigos: ¿quién escribirá una historia de la Escuela Española de verano de Middlebury? Aquel precioso pueblo del nórdico estado de Vermont, entre suaves colinas cubiertas de hierba, bajo una luz apacible y transparente, con remotos montes como telón de fondo, fue un lugar de encuentro privilegiado para los españoles, sus familiares y sus discípulos. [...] Recordar quiénes dieron clases ahí sería hacer la lista del mejor exilio, cuyas filas engrosaron los que antes habían sido españoles errabundos o emigrantes y ahora se convirtieron en emigrados (2006, 303; confróntese 2001, 28)

Estos cursos estaban dirigidos por Juan Centeno, persona sobre la que hay unanimidad a la hora de reconocer su gran categoría intelectual y moral, y sirvieron para agrupar a profesores como Joaquín Casalduero, Américo Castro, José Fernández Montesinos, Jorge Guillén, Ángel del Río, Pedro Salinas, etcétera. La mejor fuente para reconstruir los acontecimientos, más allá de los abundantes datos administrativos que brinda el estudio de Stephen A. Freeman sobre los cursos de idiomas de Middlebury College entre los años 1915-1970 (1990, 91-105 especialmente $)^{15}$, son las autobiografías de algunos de los alumnos que por allí pasaron y que compartieron aulas con Claudio Guillén. Así sucede con el caso de Carmen de Zulueta o Isabel García Lorca, quien afirma categóricamente: «La Escuela Española [de Middlebury College] llegó a tener un prestigio enorme y reunió a gentes de una categoría extraordinaria. En la vida de los exiliados españoles jugó un papel importantísimo, y en nuestra familia muy especialmente» $(2002,214)^{16}$.

En las citadas memorias de Jaime Salinas también se describe con detalle la vida académica y recreativa de los veranos en Middlebury incluyendo dos fotografías de Claudio Guillén (2003, 120-127, 141-142, 344, 379-182). Sobre su paso por estos cursos insiste Jaime Salinas, quien a diferencia de Claudio

15 Existe información también en las publicaciones periódicas de Middlebury, que se encuentran digitalizadas en <http://www.middlebury.edu/academics/lis/about/library_info/ special_collections/collections/archives/>.

${ }_{16}$ Véase también de Recuerdos míos, prologado, por cierto, por el mismo Claudio Guillén, otras afirmaciones de este jaez (García Lorca 2002, 214, 220, 221, 231-33), que vienen acompañadas además de algunas fotos, así como la referencia en las memorias de Carmen de Zulueta (2004, 100-101). Sobre estas cuestiones del exilio republicano en Estados Unidos, sigue siendo fundamental el estudio de Vicente Llorens, que vivió además el exilio en primera persona (la referencia a Middlebury 1976, 192-200). Claudio Guillén (2003) dedicó de manera muy sentida su discurso de ingreso a la Academia a este intelectual, con quien compartió amistad y avatares en las universidades de Colonia y Princeton, al que cabe añadir ahora los recientes estados de la cuestión sobre el exilio republicado y el hispanismo en Estados Unidos que conforman la obra colectiva Faber y Martínez Carazo (2010) o el dosier «El exilio republicano de 1939 y el hispanimso en Estados Unidos» (Larraz y López García 2016) y entre otras obras de carácter más general la monografía de Henry Kamen (2007), el volumen editado por Álvarez Barrientos (2011) y el extraordinario diccionario de López García y Aznar Soler (2017). 
Guillén no terminaba de formar parte del grupo de scholars fascinados por su padre, en las cualidades de Pedro Salinas como docente: «Era un profesor brillante, entretenido, que tenía la habilidad de relacionar la literatura con el arte y la historia, y aderezarlo todo con anécdotas de la vida cotidiana. A pesar de mi ignorancia, jamás me aburrí escuchándole, pero su talento no bastó para vencer mi resistencia a dedicar mi vida al hispanismo» $(2003,382)$. No fue el caso de Claudio Guillén, quien se fue acercando cada vez más a Pedro Salinas con el beneplácito de este, orgulloso de contar con otro estudioso en la familia ${ }^{17}$. El eminente profesor escribió una carta a Jorge Guillén ${ }^{18}$, fechada en Middlebury el 28 de julio de 1946 explicando lo siguiente sobre su discípulo Claudio Guillén:

A Claudio le veo mucho, claro, y le encuentro muy bien, perfectamente de aspecto y de salud, y muy animado y curioso de ánimo. Viene pacientemente a una de mis clases, y su gesto responde siempre con exactitud a lo que digo. Nos entendemos. Y me hace el honor de aliviar las flaquezas de mi quincuagena llevándome el cartapacio de Hepburn a clase muchos días. A la vuelta le releva Jaime de

17 Como desarrolla Jaime Salinas en sus memorias, la relación con su padre no fue muy sencilla. De alguna manera Pedro Salinas hubiera gustado que su hijo siguiera sus pasos de hispanista, al igual que Claudio Guillén, quien, por lo demás, desde niño llamaba la atención por sus aptitudes. Son muy ilustrativas estas sinceras palabras del hijo de Pedro Salinas: «Todo el mundo decía que Claudio era muy guapo; hablaba por los codos contando que estaba aprendiendo a tocar el violín, que ya había leído este o aquel libro, incluso opinaba sobre los poemas de su padre. A su lado yo me sentía insignificante, pero con el tiempo acabamos haciéndonos amigos. Él y su hermana [Teresa Guillén] son los únicos amigos de toda la vida que tengo» $(2003,23)$.

${ }^{18}$ En varias cartas de Pedro Salinas a Jorge Guillén se hace referencia a la creciente faceta de estudioso e intelectual de Claudio Guillén, como una donde explica que se está organizando unas jornadas conmemorativas del Quijote en Harvard donde va a intervenir Cernuda $(2007,1194)$, otras donde refiere complacido que Claudio Guillén le ha pedido una pieza de su teatro para representarla en el Club Románico de Harvard $(2007,1203,1211)$ o, en fin, una donde expresa su ánimo a Claudio Guillén para que emprenda la preparación de su tesis doctoral: «Me encuentro la carta de Claudio, creo que tiene razón, y toma la resolución más prudente. El doctorarse es cosa de máxima importancia en este mundo académico, y si ve el camino franco hacia el ansiado Ph.D. no debe dejarlo. Ya te figurarás lo que nos hubiese alegrado tenerlo aquí sobre todo ahora que se van Sol[ita Salinas] y Juan [Marichal]. Compañía la suya incomparable, en todos los sentidos. Y yo hubiera tenido larga ocasión de hablar con el mozo de sus cosas y las mías, y conocerle mejor» (2007, 1274-75). Por lo demás, Claudio Guillén -y Teresa Guillén- son personajes habituales de la correspondencia personal del poeta Salinas (2007), así como de su esposa Margarita Bonmatí. En la Houghton Library (Span 100.7) se encuentra una carta inédita de esta dirigida a ambos hermanos fechada sin año el 23 de junio en Duke University donde se puede apreciar claramente el trato familiar: Margarita Bonmatí invita a Claudio Guillén a que les haga una visita junto a su padre; con Teresa Guillén dialoga en la distancia sobre algunos asuntos de moda femenina. 
esta tarea, más o menos pesada según los autores que se expliquen ese día (2007, $1091)^{19}$.

En fin, para terminar de referir estos cursos de Middlebury, téngase en cuenta también que Claudio Guillén parece ser que llegó a impartir algún curso allí (Freeman 1990, 341), aunque no he localizado más información al respecto. A partir de aquí, la formación de Claudio Guillén continuó en Colonia $^{20}$ (desde allí viajó a España algunas temporadas durante sus vacaciones), lugar donde estuvo como lector dos años mientras redactaba su tesis doctoral ${ }^{21}$. De su vida en Colonia y su madurez intelectual dan buena cuenta las cartas que intercambió con Pedro Salinas.

Se trata de cuatro cartas que se conservan en el archivo de Pedro Salinas custodiado en la Houghton Library de Harvard (signatura Span 100), que he editado recientemente (Martín Ezpeleta 2017). En esta misma biblioteca se encuentran también las cartas que envió Pedro Salinas a Claudio Guillén (Span 100.4), las cuales han sido publicadas en la excelente edición de sus ópera omnia a cargo del profesor Enric Bou (Salinas 2007)22. Valga para ilustrar esta correspondencia un pasaje de la enviada el 25 de junio de 1951, donde, tras

19 En el archivo de Pedro Salinas custodiado en la Houghton Library de Harvard (signatura Span 100) se encuentran unos apuntes de Claudio Guillén tomados de una conferencia de Pedro Salinas sobre Garcilaso de la Vega impartida precisamente en un curso de verano en Middlebury (signatura Span 100.4), que ha analizado Montserrat Escarpín (2008). El propio Claudio Guillén reprodujo algunos de estos apuntes en su estudio sobre el profesor Pedro Salinas al hilo de la glosa de sus altas miras científicas, pedagógicas y humanas (2006, 129-43; confróntese también 2001, 31-35).

${ }^{20}$ Claudio Guillén (2006, 377-378) describe la anécdota de su viaje en barco -en el cual conoció al profesor José Manuel Blecua- a Colonia.

${ }^{21}$ Esta se titulo The Anatomies of Roguery. A Comparative Study in the Origins and the Nature of Picaresque Literature (fue publicada facsimilarmente mucho más tarde en 1987; el original se conserva microfilmado en los Archivos de Harvard: signatura HU 90.6370.10).

${ }_{22}$ Las cartas en cuestión (Salinas 2007, 1340-1341, 1344, 1414-1416 y 1481) están datadas el 17 de abril de 1950, el 4 de mayo de 1950, el 14 de enero de 1951 y el 10 de julio de 1951. Estas han de ser leídas en relación con las remitidas por Claudio Guillén (son respuestas unas de otras) fechadas en Cambridge el 12 de mayo de 1950, en Colonia el 25 de febrero de 1951 y el 4 de julio de 1951. Existe una cuarta carta de Claudio Guillén que es un fragmento sin data que versa sobre el proceso que Jaime Salinas debe seguir para sortear su intervención en la guerra (al final, como era el deseo de Jaime Salinas, fue a la guerra; el caso se explica en sus memorias: 2003, 163-65 y 196ss.), así como en las cartas de Pedro Salinas, contrario a la decisión de su hijo, donde llega a afirmar en carta a su hija Solita Salinas fechada en Baltimore el 27 de febrero de 1941: «Sigue el pollo [Jaime Salinas] con la insensata idea de servir con The fighting French, en vez de con el ejército americano. Todo por singer a Claudio [Guillén]; este tiene sus razones para eso, puesto que es medio francés, pero lo de Jaime sería absurdo» $(2007,876)$. 
trazar un fino retrato de la situación en Alemania después de la guerra, valora desde la distancia el estado político y cultural de Estados Unidos:

En Europa me tiene usted, Don Pedro, contento de estar aquí, por estos viejos mundos de Dios, que son los nuestros, contento también -emoción de índole negativa, como cuando se le quita a uno un peso de encima-, contento de no estar en América. No quisiera ser injusto con aquel país, ni «jeter le manche après la cognée»; lo criticamos, porque lo queremos y nos va decepcionando. Nos decepciona América desde el punto de vista de su mejor pasado, de esa tradición estupenda, protestante, pura, tan humana, la de Melville y Thoreau y Emerson, tradición que yo admiro mucho. Queda inundada por otra corriente, la de la técnica exenta de moralidad, el optimismo imbécil, el nacionalismo milochocientoscuarentayochesco, la inmoralidad del «assembly-line». Asistimos a algo como la desamericanización de América, como diría Don Juan Marichal, el sibilino. Pero quizás venga un día un reamericanizador de que la reamericanice: con ella, de todos modos, están nuestras esperanzas. El mayor peso que me quité de encima al salir de América fue sobre todo el de ese ambiente neurótico, que reina por lo menos entre la juventud de Harvard, y que no deja de tener influencia. Ahora bien, hay algo allá cuya calidad ya no se puede negar, no cabe dudas: es la vida cultural, la calidad del «êlite», de las mejores Universidades, de las revistas, del teatro etc. Aquí, por ejemplo, está todo muy retrasado y aislado a consecuencia de años de guerra, aislamiento y sobre todo censura ${ }^{23}$.

Y otro fragmento de una interesante carta remitida el 4 de julio de 1951, donde también describe el rumbo político que está tomando Alemania y otros países europeos. En concreto se reproduce lo concerniente a su opinión sobre la situación de los estudiantes y profesores en España, donde acaba de pasar una temporada:

Los estudiantes de la Universidad [de Valladolid] constituyen una generación del todo nueva: simpática - más sana que la francesa o la alemana, pues al fin y al cabo no han conocido la guerra última-, ñoña, ignorante, incapaz de autocrítica personal o española. Total, valen más como personas que como españoles y sobre todo que como europeos. [...] Luego di una vuelta de un mes por casi toda España. Todos los amigos preguntan por usted. La última generación de profesores -López Estrada, Muñoz Cortés, etc.- está bastante bien. La gente, descontenta de

${ }^{23}$ En carta a Solita Salinas y Juan Marichal datada posiblemente en febrero de 1951 escribe Pedro Salinas sobre esta carta de Claudio Guillén: «He tenido carta muy viva e inteligente de Claudio, sobre Alemania y la situación mundial. Confirma lo que se lee y oye (lo oímos en una radio de Londres, anteayer) sobre la irritación que empieza a cansarles a los europeos, la insistencia nada delicada, más bien brutal, con que Estados Unidos los empuja a los armamentos. La pintura que hace de Alemania es tremenda: destrucción, escepticismo, inmoralidad. ¡Los bienes de la guerra! Ahora se va a Valladolid a pasar un mes de vacaciones» $(2007,1430)$. Véase también la carta a Jorge Guillén fechada el 19 de marzo de 1951, donde se refiere en los mismos términos a esta carta de Claudio Guillén (2007, 1441). 
un modo sordo, alarmante. Todos tienen miedo, verdadero miedo de una posible guerra civil. No creo que por ahora el factor contrario -el descontento creciente, que alcanza cada día sectores más amplios de la población, según van sintiendo personalmente y concretametnte el aprieto- sea más fuerte. El gobierno intenta realizar reformas u obras nuevas, pero son -como el «Talgo», un tren nuevo entre Irún y Madrid, superior a cualquiera que haya visto en América- los gritos del afónico. Por ahora no se ve el final del túnel24.

El siguiente epígrafe aborda ya la labor docente de Claudio Guillén.

\section{Claudio Guillen, Profesor en Estados Unidos}

No tardó mucho Claudio Guillén en poder ejercer de profesor. Ya ha quedado referido su trabajo durante dos años en Colonia. Después viajó de nuevo a Estados Unidos, donde defendió su tesis en 1953. Recién doctorado por Harvard, consiguió un puesto en 1954 en la Universidad de Princeton. Años más tarde se trasladó a La Jolla, la Universidad de California en San Diego, donde impartió clases desde 1965 a 1976. Y luego vivió una temporada en España hasta 1978. Fue entonces cuando, como informa la correspondencia intercambiada con Harry Levin ${ }^{25}$, su profesor y amigo hasta el final de sus días,

${ }^{24}$ La relación con su «profesor amigo» Pedro Salinas, como queda explicado, fue mucho más allá de lo académico. El hecho de que una obra tan decisiva en la vida de un profesor como su tesis doctoral vaya dedicada a la memoria de Pedro Salinas no es un dato insignificante. Por lo demás, en el fondo de Pedro Salinas de la Houghton Library (Span 100) se conserva el siguiente telegrama a Juan Augusto Marichal: "Abrazos de vuestro hermano=Claudio», cuya fecha del 5 de diciembre de 1951 nos indica que se trata de una nota de pésame por el reciente fallecimiento de Pedro Salinas. Ya el 12 de octubre había enviado una postal (de la Plaza Mayor de Salamanca) firmada por Manuel García Blanco, su padre Jorge Guillén -en caligrafía de Claudio Guillén- y Claudio Guillén a Pedro Salinas deseándole su pronta recuperación (Houghton Library, Span 100). Lo mismo cabe decirse de otra carta del 9 de octubre firmada por varios de los amigos de Salinas en España: Aleixandre, Díez Crespo, Fernández Almagro, Dámaso Alonso, Bleiberg, Llosent, Cossío, Ridruejo, Rosales, Bousoño, Jorge Guillén y Claudio Guillén (Houghton Library, Span 100).

25 Estas cartas escritas en inglés forman parte del fondo Harry Levin, custodiado en la Houghton Library de Harvard (signatura Am 2461 y Stor 342). Se trata de cuatro carpetas con abundantes cartas de tema profesional principalmente (fechadas desde marzo de 1956 hasta abril de 1992, cuando Harry Levin ya se encuentra convaleciente; falleció en 1994) de Harry Levin a Claudio Guillén y unas pocas de este al primero (también hay alguna otra carta profesional sin especial relevancia de Claudio Guillén a otros profesores como Harold C. Martin, Bruton Pike y una de Morton Wilfred Blommfield dirigida a Claudio Guillén). Entre los asuntos profesionales que se tratan destacan las noticias sobre la preparación de artículos y libros (por ejemplo, Guillén habla de sus estudios sobre el exilio, de su artículo sobre Américo Castro y Sánchez Albornoz o del texto sobre Salinas que prepara para su efemérides), la organización de congresos (es relavante el primer congreso de Literatura 
el americano le filtró en una carta fechada el 12 de diciembre de 1975 la noticia de su candidatura a un puesto en el Departamento de Literatura Comparada en la Universidad de Harvard. Era, hecha la excepción de un seminario impartido por Claudio Guillén en Harvard en la primavera del curso 1957-1958 titulado «Lyric Poetry from Garcilaso to Quevedo», su reencuentro con esta institución, cuyos avatares, no obstante, conocía perfectamente por su correspondencia con Harry Levin.

Dudó mucho Claudio Guillén en aceptar el puesto e incluso llegó a declinarlo, pese a la decepción de Harry Levin, quien le animó encarecidamente a que fuera a Harvard. Entre otras razones, le seducía la posibilidad de volver a España (en carta fechada el 4 de marzo de 1977 explica Guillén que la Universidad de Málaga le ha invitado a ser un año profesor de Literatura Comparada), así como la existencia de una importante oferta profesional de otra universidad del Este de Estados Unidos. Además, en otra ocasión Claudio Guillén se pregunta si el Departamento de Literatura Comparada de Harvard, que tanto cariño y respeto le merecen, no necesitaría a alguien más joven que él. Ya en carta fechada el 6 de junio de 1978 y con membrete de la Universidad de Málaga afirma que cada día tiene más deseos de ir a Harvard. Efectivamente, en 1978 se trasladó a esta institución, donde compaginó sus numerosos viajes a España con la tarea docente en los departamentos de Literatura Comparada y Lenguas y Literaturas Romances hasta 1988. Del primero llegó a ser director durante los años 1979-1982 (lo relevó Walter Kaiser) y en 1983 sucedió a Harry Levin en la cátedra que lleva su nombre. Su labor fue merecedora también de la distinción de profesor emérito a partir de 1988. Veamos ahora con algo de detalle su trabajo en Harvard repasando las asignaturas que allí impartió.

Pues bien, en el curso 1978-1979 Claudio Guillén entró a formar parte de la plantilla de profesores del Departamento de Literatura Comparada. Sus primeras asignaturas fueron francamente ambiciosas y toda una demostración de su competencia, como la dedicada a Cervantes y la tradición novelística, donde comentaba con detalle el Quijote, pero también otras novelas de grandes autores como Fielding, Stendhal, Zola, Dostoievsky y Thomas Mann; con es-

\footnotetext{
Comparada que Guillén organizó en España en mayo de 1974) o, en fin, las presentaciones y recomendaciones de profesores (Claudio Guillén, por ejemplo, pone en contacto al profesor Francisco Rico con Harry Levin, a quien el catedrático de la Universidad de Barcelona desea pedir un prólogo para uno de sus libros). Sobre esta correspondencia y la relación entre Claudio Guillén y Harry Levin, es fundamental el trabajo de Andrés Pérez-Simón (2014). Especialmente interesante resulta la información relativa al denominado «Informe Levin» que este preparó (Levin 1995) para la recientemente fundada American Comparative Literature Association (ACLA). Este informe supone un estado de la cuestión del comparatismo norteamericano en 1965, que Claudio Guillén tomó como referencia para impulsar un prograna de doctorado de Literatura Comparada en la Universidad de California en San Diego (Pérez-Simón 2014, 301-303).
} 
pecial atención, como se señala en el cuadernillo de asignaturas de la Facultad de Artes y Ciencias de ese curso (Official Register of Harvard University 1978, 214), a la función de las ideas y el papel del diálogo en las novelas. Al año siguiente se vio obligado a reducir ligeramente la asignatura, eliminando las obras de Zola y Thomas Mann (Official Register of Harvard University 1979, 243), tal y como hizo también en el siguiente curso de 1980-1981. Esta asignatura estaba dirigida no solo para los alumnos del Departamento de Literatura Comparada, sino también para los del Departamento de Lenguas y Literaturas Romances.

La segunda asignatura que comenzó impartiendo en 1978-1979 fue un curso sobre la literatura del exilio, cuyo programa era el que sigue: «Prevalent thematic responses to the experience of exile will be discussed, with the reference to changing social circumstances, amog writers form Ancient Rome, China, medieval Italy, the Renaissance, and the Romantic movement» (Official Register of Harvard University, 1978, 219). Esta asignatura también se consolidó en el programa de doctorado del Departamento de Literatura Comparada impartiéndose los tres siguientes cursos. No es difícil adivinar que detrás de estas clases está su importante El sol de los desterrados. Literatura y exilio, que publicaría por primera vez en un congreso americano en 1990.

Y la tercera asignatura con que comenzó su andadura en Harvard fue un seminario introductorio sobre «Critical Methods», donde repasaba los procedimientos y propuestas de Croce, Lukács, Wölfflin, T.S. Eliot, Valéry, Spitzer, Curtius, Gramsci, Poulet, Barthes y Jause en sus textos (Official Register of Harvard University 1978, 221). Esta asignatura como tal no tuvo continuidad los siguientes años (reaparece en el curso de 1987-1988), aunque, en la misma línea teórico-literaria, encontramos en 1980-1981 un curso monográfico sobre una de las corrientes críticas que más interesó a Claudio Guillén, la Estilística (que analizó en varios trabajos: en el prólogo a los estudios de Francisco García Lorca, en Teorías de la historia literaria...): «Methods of Stylistic Analysis: An examination of changing approaches to the function of style, from Spitzer to Riffaterre» (Official Register of Harvard University 1980, 260). Esta se mantuvo (con una ligera modificación que afecta solo a la descripción de la asignatura, donde incluye explícitamente los nombres de Dámaso Alonso y Barthes, Official Register of Harvard University 1981, 251) hasta 1986-1987, es decir, prácticamente todos los años que Claudio Guillén estuvo en Harvard.

Algo muy parecido sucedió con otra asignatura nueva que impartió desde 1979-1980 -su segundo curso en Harvard- hasta 1986-1987, la también muy ambiciosa «Theories of Literary Form», que analizaba las ideas de Aristóteles, las Poéticas del Renacimiento italiano, el Neoclasicismo y las nociones de F. Schelegel, Coleridge, los formalistas rusos y los críticos franceses y suizos sobre la forma literaria (Official Register of Harvard University 1979, 250). En 1980-1981, eso sí, se recondujo ligeramente la asignatura -ahora intitualda «Theories of Literary Genre»-, que repasaba: «Conceptions of the nature of 
genre, and modes of classification, will be studied in Aristotle, Quintilian, Renaissance Italian critics, Schiller, Goethe, Brunetière, Croce, Tynyanov, Genette, and others» (Official Register of Harvard University 1980, 260).

En su segundo curso en Harvard Claudio Guillén estrenó otra asignatura «The Picaresque Tradition: Rogues, Vagabonds, Marginal Men: Commentaries on Lazarillo de Tormes and its successors - narratives by Quevedo, Le Sage, Defoe, Smollet, Dickens, Thomas Mann, Bellow, and Günter Grass, with special attention to the myth of the half-outsider, critical views of society, and the uses of the first-person voice» (Official Register of Harvard University 1980, 252). Un completo curso donde, como se habrá notado, aparecen autores claves agrupados en torno a la noción de picaresque. Este curso tan cercano a la primera etapa de la trayectoria investigadora de Claudio Guillén, a su tesis doctoral, se mantuvo tres cursos académicos en los programas de Harvard y se ofertó también para el Departamento de Lenguas y Literaturas Romances.

En el marco de este departamento precisamente se sitúa otra asignatura nueva -se impartió los dos siguientes cursos también- sobre la narrativa española del XIX, que presentaba conceptos básicos como costumbrismo al lado de la descripción de la situación socio-cultural de lo que él llama «el nuevo ciclo novelístico español» (autores como Mesonero Romanos, Alarcón, Valera, Galdós, Clarín y Pardo Bazán; sobre estos tres últimos publicó inteligentes trabajos unos años después [Official Register of Harvard University 1980, 714]). No fue este el único curso dirigido a los alumnos del Departamento de Lenguas y Literaturas Romances.

En 1982-1983 impartió dos seminarios específicos más. Uno titulado «Literary Criticism: Stylistics. A consideration of the Hispanic tradition of style studies, in both Spain and Latin America, and of recent critiques of the procedures and purposes that such studies implied» (Official Register of Harvard University 1982, 736), que repitió en 1985-1986; y un segundo sobre la poesía moderna española, que también estuvo presente en los cursos de 1985-1986 y 1986-1987. Este último responde a otra de sus líneas de investigación más queridas, la poesía de autores como Pedro Salinas, Federico García Lorca o Rafael Alberti sobre los que publicó diversos estudios. En 1986-1987 hizo lo propio con dos nuevos seminarios: «Cervantes: Cervantes' major works (exlusive of Don Quixote)» (Official Register of Harvard University 1986, 663), que también impartió en 1987-1988; y «Garcilaso, Fray Luis, Quevedo: A study of the major poems, with reference to the genre of system of their times» (1986, 666). En fin, autores que conocía muy bien, tal y como lo demuestra su El Primer Siglo de Oro, que apareció por aquellas fechas (1988).

Por último, en 1984-1985 se encargó de una nueva asignatura dirigida a los alumnos del Departamento de Literatura Comparada: «Literary Theory: Pro-Seminar. Categories of Comparative Criticism: Genres, Forms, Themes, International Relations, and Literary History» (Official Register of Harvard University 1984, 182), que repitió hasta el curso de 1986-1987. Como reconocerá el 
lector de Claudio Guillén, la descripción de este curso repite casi a la letra el índice de Entre lo uno y lo diverso (1985), cuya redacción estaba ultimando por aquel entonces. Por lo demás, se trataba de asuntos presentes desde sus primeras clases en Harvard y sobre los que pensó mucho y escribió no menos páginas, que hoy se puede recuperar bajo títulos ya clásicos como Literature as System (1971), Teorías de la historia literaria (1989), The Challenge of Comparative Literature (1993) o Múltiples moradas (1998), entre otros ${ }^{26}$.

Como es sabido, en 1982 Claudio Guillén se trasladó a la Universidad de Barcelona, aunque compaginó su trabajo en España con algunos cursos en Harvard, según se ha explicado. El último que impartió en esta Universidad fue el 6 de mayo de 1992, cuando ya jubilado (se jubiló en 1989) le encargaron pronunciar la conferencia de Renato Poggioli, lo que explica en carta a Harry Levin fechada el 20 de abril de 1992 (con membrete del Dept. of Spanish, Vanderbilt University, Nashville, Tenn.) que le trae muchos recuerdos y que significa un «improbable and marvelous event for me, circular beyond words» (Harry Levin, en su última carta, fechada el 24 de abril de 1992, retoma la idea del «cyclical gesture of some purport»). Aprovechó este viaje a Harvard para visitar la estupenda Biblioteca Widener, pues, como explica a Levin, trabajaba jubilado con mayor placer que nunca (especialmente en su casa cerca de Nerja). En su última carta a Harry Levin (20 de abril de 1992) Claudio Guillén escribe a este respecto: «I am still doing Comparative Literature, very stubbornly, very loyally with respect to my origins. [...] I feel proud of all that I was able to do and achieve for the department at Harvard while I was there. Not to mention the lot concentration and other things»».

En este punto, como unos años más tarde escribió, Claudio Guillén tenía claro que en España había que apostar por un modelo como el del Departamento de Literatura Comparada de Harvard, que tan bien conocía. Con todo, en aquella temprana época para la Literatura Comparada española, explica en Entre lo uno y lo diverso que no tuvo mucho éxito:

La voz de la Literatura Comparada se sigue oyendo en aquellos círculos donde las actitudes y los valores que expresa son ya una tradición respetada. Pienso en las grandes universidades norteamericanas, con sus departamentos de Literatura Comparada [...] En Harvard trabaja con valentía, [...] un departamento minoritario, pequeño -cierto que nunca fue grande, tampoco en mis tiempos, los de Harry Levin-, pero que reúne a alumnos muy buenos, y que sigue poniendo en práctica unos esquemas docentes de probada eficacia. (En alguna ocasión he

\footnotetext{
${ }^{26}$ Véase ahora sobre la relevancia de los estudios de Claudio Guillén los tempranos trabajos de Darío Villanueva (1999) y Marc Zimmerman (2005) y otros posteriores como García Jurado, Reader y Villar (2009), Cots y Monegal (2010) y Cabo Aseguinolaza (2007), donde se incluye un interesante análisis del pensamiento literario de Claudio Guillén en el contexto del comparatismo norteamericano.
} 
tratado de describirlos y explicarlos a mis colegas españoles, claro que inútilmente) $(2005,13)$.

Finalmente, hay que apuntar muy brevemente que Claudio Guillén no solo fue influido por la investigación en Harvard, sino también por su organización política. El autor de Literature as System destacó por escrito cómo la organización de la Universidad privada de Harvard era un modelo muy interesante para intentar reproducir en España, así como su imbricación en un sistema competitivo entre universidades completamente autónomas:

Se trata de desburocratizar y de descentralizar. Sobre todo descentralizar, a los más altos niveles. Antes de explicarme, dejadme que os diga algo de mi experiencia en Estados Unidos, en la universidad de Princeton, la de California y sobre todo la de Harvard, donde fui catedrático y jefe de Departamento durante bastantes años. Buen número de universidades de primera fila son privadas y no reciben directriz alguna de ningún Ministerio de Washington. [...] En realidad la emulación conduce a que cualquier innovación por parte de una de esas entidades estimule el análisis y frecuentemente la imitación por parte de las demás. En Harvard todas las cuestiones científicas y pedagógicas principales están en manos del Decano [...] Aquí en España urge descentralizar la enseñanza universitaria, basándose en un hecho fundamental, la existencia de una estructura política poco menos que federal, la del Estado de las Autonomías. [...] Comprendo que esto significa exigirles mucha audacia, no menos imaginación y, lo que más escasea, confianza en sus paisanos. Pero con un grado módico de las tres basta para poder concebir una relación de competencia, aprendizaje y observación mutuas, comparable a la de Stanford y Harvard $(2001,64-65)^{27}$.

\section{EN LAS PRIMERAS PÁGINAS DEL COMPARATISMO ESPAÑOL}

A lo largo de este trabajo se ha reconstruido la historia de Claudio Guillén en la Universidad de Harvard (y Middlebury), que no solo supone parte de la extraordinaria trayectoria profesional y personal de un eminente estudioso; sino también un impulso determinante para el comparatismo español. Y es que los trabajos de Claudio Guillén realizados en Estados Unidos fueron leídos con fruición por jóvenes profesores como Darío Villanueva o José María Pozuelo Yvancos. Estos autores fueron en buena medida los encargados de consolidar el comparatismo en España, que no hubiera sido posible sin ese diálogo creador -que diría Pedro Salinas- que se estableció mezclando, de un lado, los frescos

27 En las siguientes páginas, Claudio Guillén (2001, 66-67) desarrolla y reivindica para la universidad española el Dean's Committee de Harvard, conjunto de especialistas en diferentes disciplinas que se reúnen con el Decano dos veces cada mes para discutir con rigor sobre los asuntos del momento. 
estudios de Claudio Guillén que asimilaban los planteamientos teórico-metodológicos de grandes pensadores en Estados Unidos, como sus maestros en Harvard Harry Levin y Renato Poggioli, pero también René Wellek o Northrop Frye. Y de otro, las reflexiones de algunos teóricos europeos, como por ejemplo el rumano afincado en España Alejandro Cioranescu o el portugués Vítor Manuel Aguiar y Silva ${ }^{28}$, así como los tanteos comparatistas de eminentes filólogos españoles de la primera mitad del siglo XX.

Entre estos, hay que incluir a autores como Ángel Valbuena Prat, que, además de colaborar con Paul Van Tieghem en su famoso manual La literatura comparada de 1931 y en el Repertorio cronológico de las literaturas modernas de 1937, realizó acercamientos comparatistas de gran mérito en sus manuales. También contribuyeron decisivamente a desbrozar un camino comparatista dos obras muy relevantes, a despecho de no estar completamente apuradas, como fueron la Historia de la literatura universal de Martín de Riquer y José María Valverde (1957), que superaba alguna anterior como la Historia de la literatura universal coordinada por Ciriaco Pérez Bustamante (1946), y la Historia general de las literaturas hispánicas dirigida por Guillermo Díaz-Plaja (1969-1973), que podríamos considerar que retoma y cierra al mismo tiempo la etapa del Centro de Estudios Históricos dirigido por Ramón Menéndez Pidal.

Este fin de etapa viene casi a coincidir en el tiempo con un hito para el comparatismo español como fue la celebración del «Primer Coloquio de Literatura Comparada» en 1974, entre cuyos organizadores se encontraba Claudio Guillén. Pero el autor de Entre lo uno y lo diverso también participó en la fundación tres años más tarde de una Sociedad Española de Literatura General y Comparada (SELGYC), que presidió desde 1984 hasta 1990 para luego ser nombrado presidente de honor. En el decimoctavo simposio de la Sociedad celebrado en 2008 se le rindió un importante homenaje, donde no faltaron testimonios de colegas y discípulos que reconocieron la relevancia de su magisterio y esa labor -que él vivió como fallida- de consolidar la Literatura Comparada en España (Cots y Monegal 2010).

Sin embargo, nada de esto hubiera sucedido sin esa historia de Claudio Guillén en Harvard, una historia de cariño y aprovechamiento mutuo que entrelaza claramente lo estrictamente profesional con lo personal, y que hermana instituciones y países. La narración de esta historia que aquí se ha llevado a cabo muestra inequívocamente cómo Claudio Guillén adquirió gran parte de su

${ }^{28}$ Sobre el origen del comparatismo, véase ahora el completo estudio de Maestro (2008), que revisa todos los hitos europeos que conformaron esta disciplina. Confróntese también el reciente resumen de Fernando Cabo Aseguinolaza (2017), que destaca el debate llevado a cabo en la otra orilla del Atlántico, con impulsores de la disciplina como Horst Frenz y Newton P. Stallknecht, claves en el pensamiento de Claudio Guillén y otros comparatistas de su generación, como el francés René Étiemble. 
identidad como comparatista en Harvard, donde antes de transmitir sus conocimientos como profesor gestó sus líneas de investigación más importantes como estudiante. Así, la documentación traída a colación sobre su formación en esta institución y sus primeros pasos como profesor resulta fundamental para entender el origen de la Historia del comparatismo en España, en cuyas primeras páginas -y en alguna más- figura Claudio Guillén. Estas son, para terminar, sus palabras de discípulo y maestro ejemplar:

\begin{abstract}
Alguna vez me he preguntado si nuestros maestros no fueron demasiado buenos, si no fue excesivo el peso de su ejemplaridad. Quienes tuvimos aquella preparación y nos doctoramos en aquellas aulas, seguimos nuestros caminos singulares y vivimos nuestras vidas de profesores por separado, bien que mal, o mejor que pudimos y supimos. De mí sé decir que todos mis trabajos se me aparecen en el mejor de los casos como un esfuerzo por estar a la altura de mi memoria, de mi deuda con aquellos hombres admirables; y sobre todo con los valores y las cualidades que, más allá de toda identidad individual, ellos supieron convertir en vida real, solidaria y perdurable $(2001,35)$.
\end{abstract}

\title{
FUENTES
}

Annual reports of the President and Treasurer of Harvard College. 1890-91. Massachusetts: Harvard University, 1892.

Escarpín Gual, Montserrat. 2008. «Garcilaso de la Vega y Pedro Salinas: la voz a ti debida». Revista de literatura LXX, 140: 553-575. https://doi.org/10.3989/revliteratura.2008.v70. i1 140.70

García Lorca, Francisco. 1984. De Garcilaso a Lorca. Editado y prologado por Claudio Guillén. Madrid: Istmo.

García Lorca, Isabel. 2002. Recuerdos míos. Editado por Ana Gurruchaga; prólogo de Claudio Guillén. Barcelona: Tusquets.

Gilman, Stephen. 2002. Del Arcipreste de Hita a Pedro Salinas. Traducido por Elena Abós; prólogo de Francisco Márquez Villanueva y Claudio Guillén. Salamanca: Universidad de Salamanca.

Guillén, Claudio. 2001. Entre el saber y el conocer. Moradas del estudio literario. Valladolid: Fundación Jorge Guillén.

Guillén, Claudio. 2003. De la continuidad. Tiempos de historia y de cultura. Discurso leido el día 2 de febrero de 2003 en su recepción pública, por el Excmo. Sr. Don Claudio Guillén y contestación del Excmo. Sr. Don Francisco Rico. Madrid: Real Academia Española.

Guillén, Claudio. 2005. Entre lo uno y lo diverso. Introducción a la Literatura Comparada. (Ayer y hoy). Barcelona: Tusquets.

Guillén, Claudio. 2006. De leyendas y lecciones. Siglos XIX, XX y XXI. Barcelona: Crítica.

Guillén, Jorge y Pedro Salinas. 1992. Correspondencia (1923-1951). Edición, introducción y notas de Andrés Soria Olmedo. Barcelona: Tusquets.

Harvard University Archives. Archives accession 17478, HU 30.10, HU 90.6370.10, HUC 8500.16, HUE 22.153.75. 
Houghton Library. Span 100, Am 2461, Stor 342.

Martín Ezpeleta, Antonio. 2017. «Cartas inéditas de Claudio Guillén a Pedro Salinas». En Claudio Guillén en el recuerdo, ed. Antonio Monegal, Enric Bou y Montserrat Cots, 125-135. Venecia: Biblioteca di Rassegna iberistica.

Official Register of Harvard University. Final Announcement of the Courses of Instruction Offered by the Faculty of Arts and Sciences... Massachusetts: Harvard University, 19461949, 1978-1987.

Pérez-Simón, Andrés. 2014. "A personal history of the "American hour" of Comparative Literature. Claudio Guillén in conversation with Harry Levin». 1616. Anuario de literatura comparada IV: 295-312.

Report of the President of Harvard College and reports of departments... Massachusetts: Harvard University, 1948-1949.

Salinas, Jaime. 2003. Travesías. Memorias (1925-1955). Barcelona: Tusquets.

Salinas, Pedro. 2007. Obras Completas III. Epistolario. Edición de Enric Bou; prólogo de Andrés Soria Olmedo. Madrid: Cátedra.

Williams College Archives \& Special Collections.

Zulueta, Carmen. 2004. Caminos de España y América. Prólogo de Isaías Lerner y Alfonso Armada. Madrid: Publicaciones de la Residencia de Estudiantes.

\section{REFERENCIAS BIBLIOGRÁFICAS}

Álvarez Barrientos, Joaquín, ed. 2011. Memoria del hispanismo. Miradas sobre la cultura española. Madrid: Siglo XXI.

Cabo Aseguinolaza, Fernando. 2007. «Claudio Guillén o los equívocos de la teoría». En Pensamiento literario española del siglo XX, ed. Túa Blesa, I, 74-89. Zaragoza: Prensas Universitarias.

Cabo Aseguinolaza, Fernando. 2017. «Notas para unha voz de dicionario: literatura comparada». En Sobre letras e signos. Estudos en homenaxe a Anxo Tarrio Varela, ed. Armando Requeixo Cuba, 205-218. Santiago de Compostela: Xunta de Galicia.

Cots, Montserrat y Antonio Monegal, eds. 2010. Actas del XVII Simposio de la Sociedad Española de Literatura General y Comparada. Madrid: SELGYC.

Faber, Sebastiaan y Cristina Martínez Carazo, eds. 2010. Contra el olvido. El exilio español en Estados Unidos. Alcalá de Henares: Universidad de Alcalá.

Freeman, Stephen Albert. 1990. The Middlebury College Foreign Language Schools. The Story of a Unique Idea. Middlebury: Middlebury College.

García Jurado, Francisco, Margit Reader y Juan Felipe Villar Dégano, eds. 2009. Claudio Guillén, lecciones de un maestro. Madrid: Universidad Complutense.

Kamen, Henry. 2007. The Disinherited. The Exiles Who Created Spanish Culture. Westminster: Allen Lane.

Larraz, Fernando y José-Ramón López García, coords. 2016. «El exilio republicano de 1939 y el hispanismo en Estados Unidos». Laberintos. Revista de estudios sobre los exilios culturales españoles 18: 111-280.

Levin, Harry. 1994. «Comparative Literature at Harvard». En Building a Profession. Aubiographical Perspectives on the History of Comparative Literature in the United States, ed. Lionel Gossman y Mihai I. Spariosu, 13-23. Albany: State University of New York. 
Levin, Harry. 1995. «The Levin Report, 1965». En Comparative Literature in the Age of Multiculturalism, ed. Charles Bernheimer, 13-23. Baltimore - Londres: Johns Hopkins University Press.

Llorens, Vicente. 1976. El exilio español de 1939. I. La emigración republicana. Dir. José Luis Abellán. Madrid: Taurus.

López García, José Ramón y Manuel Aznar Soler, eds. 2017. Diccionario biobibliográfico de los escritores, editoriales y revistas del exilio republicano de 1939. 4 vols. Sevilla: Renacimiento.

Maestro, Jesús G. 2008. Idea, concepto y método de la Literatura Comparada. Desde el Materialismo Filosófico como teoría literaria contemporánea. Vigo: Academia del Hispanismo.

Monegal, Antonio, Enric Bou y Montserrat Cots, eds. 2017. Claudio Guillén en el recuerdo. Venecia: Biblioteca di Rassegna iberistica.

Villanueva, Darío. 1999. "Claudio Guillén: la Literatura Comparada en y desde España». En Sin Fronteras. Ensayos de Literatura Comparada en Homenaje a Claudio Guillén, ed. Darío Villanueva, coords. Antonio Monegal y Enric Bou, 13-19. Madrid: Castalia - Universidad Pompeu Fabra - Universidad de Santiago de Compostela.

Zimmerman, Marc. 2005. "Claudio Guillén and the theory of literary systems». Pre-post Positions. Essays on Structures \& History, Literary Theory \& Aesthetics, 83-105. Houston: Global Casa-Lacasa.

Fecha de recepción: 07 de octubre de 2017.

Fecha de aceptación: 28 de noviembre de 2017. 